Service social

\title{
Le partenariat de recherche. Éléments de définition et ancrage dans quelques études de cas
}

\section{Michèle Clément, Francine Ouellet, Laurette Coulombe, Caroline Côté et Lucie Bélanger}

Volume 44, numéro 2, 1995

Visages de la violence

URI : https://id.erudit.org/iderudit/706697ar

DOI : https://doi.org/10.7202/706697ar

Aller au sommaire du numéro

Éditeur(s)

École de service social de l'Université Laval

ISSN

1708-1734 (numérique)

Découvrir la revue

Citer cet article

Clément, M., Ouellet, F., Coulombe, L., Côté, C. \& Bélanger, L. (1995). Le partenariat de recherche. Éléments de définition et ancrage dans quelques études de cas. Service social, 44(2), 147-164. https://doi.org/10.7202/706697ar
Résumé de l'article

Cet article présente une recension des écrits sur la question du partenariat de recherche. Les auteures définissent d'abord cette pratique relativement récente, puis la documentent sous l'angle des conditions de réussite, des obstacles ainsi que des défis qu'elle pose. Des analyses d'expériences de partenariat entre groupes de femmes et chercheuses universitaires viennent corroborer ou nuancer, selon le cas, les propos recensés dans les écrits théoriques. 


\title{
Le partenariat de recherche: éléments de définition et ancrage dans quelques études de cas
}

\author{
Michèle CLÉMENT ${ }^{1}$ \\ Francine OUELLET \\ Laurette COULOMBE \\ Caroline CÔTÉ \\ Lucie BÉLANGER
}

Centre de recherche interdisciplinaire sur la violence familiale et la violence faite aux femmes (CRI-VIFF)

Le CRI-VIFF est un centre de recherche interuniversitaire, multidisciplinaire et partenarial. Dans son premier rapport annuel (1992-1993), il est dit: «...ce qui particularise le CRI-VIFF, ce sont ses activités de recherche entièrement intégrées à l'action des organismes du milieu ; la recherche y est pragmatique, non seulement parce qu'elle a des retombées concrètes, mais aussi parce qu'elle est pensée, définie, élaborée et réalisée en étroite collaboration avec les milieux de pratique»(Clément, 1993: 2). 
Mais qu'en est-il de ce partenariat? Comment s'exprime-t-il ? Comment, de part et d'autre, est-il défini? Quelles difficultés sont rencontrées? Et, enfin, quels sont les critères qui garantissent la réussite de cette démarche?

Pour trouver des réponses à ces questions, I'Assemblée des partenaires du CRI-VIFF a émis la résolution de consacrer chaque année une partie de son budget à la thématique du partenariat. Cette volonté s'est concrétisée, notamment, par l'examen des écrits sur le sujet ainsi que par quelques études de cas de partenariat de recherche entre les groupes communautaires et le milieu universitaire ${ }^{2}$. $C^{\prime}$ est le fruit de cette première étape qui est présenté ici ${ }^{3}$.

D'entrée de jeu, il faut dire que, bien que plusieurs auteurs reconnaissent l'importance de la collaboration entre chercheurs et praticiens et ce, depuis les années 80 surtout, peu d'études comme telles ont porté sur le partenariat. Celles qui ont été repérées sont par ailleurs très récentes (début des années 90).

Dans le cadre de la présente recension des écrits, la question des définitions données au partenariat a été privilégiée, de même que les conditions de sa réussite et les obstacles à sa réalisation. Les articles qui ont servi à cette recension proviennent de divers secteurs de l'intervention et de la recherche et positionnent leur partenariat à partir de bases parfois fort différentes. À titre d'exemple, on peut parler de partenariat entre des universitaires ou des institutions publiques, parapubliques ou des organismes ou groupes communautaires. Il y a donc lieu de penser que la notion même de partenariat sera colorée de ces contextes, aussi différents soient-ils. Toutefois, il nous apparaissait important de tenir compte de l'ensemble de cette littérature sur le plan théorique. Par ailleurs, pour ce qui est des expériences de partenariat étudiées, celles-ci font référence uniquement à des démarches communes entre groupes de femmes et milieux universitaires. Elles sont présentées à titre d'études de cas, dans le but de documenter les acquis théoriques.

\section{DÉFINITIONS DU PARTENARIAT}

Tout d'abord, l'examen attentif des écrits amène un premier constat, soit I'emploi synonymique des termes «collaboration» et "partenariat». En effet, certains auteurs, Bogo et al. (1992), Epstein et Kohn (1992) et Galinski et al. (1993) notamment, utilisent le mot "partenariat» pour parler en réalité de collaboration entre chercheurs et praticiens; "collaboration» est parfois synonyme de "partenariat» 
pour tel auteur et les termes sont alors interchangés dans le texte. Parfois même, une recherche-action est considérée comme du partenariat. La confusion que cette synonymie engendre est significative quant au travail de distinction à établir.

Ainsi, pour éviter toute ambiguïté, il devient nécessaire de distinguer dès le départ "partenariat» et "collaboration ». Le Center for Research on Violence against Women and Children ${ }^{4}$ introduit à ce sujet une nuance intéressante. Pour ce centre, il y a en effet collaboration entre deux parties; il est question de collaboration lorsqu'il y a coopération à une étape ou à plusieurs étapes de la recherche et ce, le plus souvent à un stade avancé de celle-ci, alors que le partenariat comprend au contraire une coopération à toutes les étapes de la recherche. Cette distinction sera retenue dans les suites de ce texte.

Dans son document de réflexion réalisé dans le cadre d'un symposium organisé par The Provincial Partnership Committee on Family Violence (Saskatchewan), Adams et al. (1994: 1) définissent le partenariat comme étant un rassemblement de "personnes qui travaillent ensemble en maintenant des rapports personnels égaux ${ }^{5}$ » (Adams et al., 1994: 1). Pour eux, le partenariat est atteint lorsque I'ensemble des individus écoutent, partagent et respectent chaque opinion, les connaissances et les différences réciproques. Bogo et ses collaborateurs (1992: 233) vont encore plus loin en ajoutant que dans leur "modèle de collaboration » tous les participants doivent être engagés dans une activité collégiale et ce, dès le début de la recherche. Cet élément est repris par Ouellet (1994: 3) pour qui les partenaires doivent se considérer comme égaux et complémentaires dans leur «collaboration».

Dans sa tentative de définir ce qu'est un partenariat féministe de recherche entre des militantes autochtones et des universitaires, Labrecque (1994: 1-2) décrit celui-ci comme étant un outil d'empowerment (pleins pouvoirs) qui «ne concerne pas seulement la construction du sens à partir de l'environnement de la recherche mais aussi la relation entre les partenaires. Cette relation entre les partenaires se caractérise par une existence préalable au processus de recherche même ». Labrecque ajoute que, dans ce contexte, l'idée de partenariat est «hybride», connotant «à la fois le constructivisme, la pratique terrain et la recherche participative».

Reconnaissant I'ambiguïté du terme "partenariat», certains auteurs québécois préfèrent parler $d^{\prime}$ " interface», à savoir « un espace social dans lequel des acteurs aux agendas variés se déploient» (Couillard et Côté, 1993: 30). Pour sa part, dans une typologie des thèses à propos des liens entre la recherche et la pratique, Groulx 
(1994: 45) parle de l' « interface recherche-pratique» qui passe par la réappropriation par le milieu de la pratique des catégories et des instruments de la recherche. Pour Deslauriers et Pilon (1994:31), le partenariat désigne davantage un vœu pieux qu'une réalité. Ces auteurs reconnaissent toutefois que le mot traduit un courant qui s'amplifie et désigne une approche faite de négociations et de coopérations conflictuelles.

La dimension de la négociation apparaît aussi dans la définition du partenariat donnée par Panet-Raymond et Bourque (1991: 9-10). Dans leur recherche-action évaluative portant sur les relations entre les organismes communautaires et les établissements publics (CLSC), ils le définissent ainsi :

un rapport égalitaire et équitable entre deux parties différentes par leur nature, leur mission, leurs activités, leurs ressources et leur mode de fonctionnement. Dans ce rapport, les deux parties ont des contributions différentes mais jugées mutuellement comme également essentielles. Le partenariat réel est donc fondé sur un respect et une reconnaissance mutuelle des contributions et des parties impliquées dans un rapport d'interdépendance.

Dans ce contexte, I'objet du partenariat devient « un échange de services et/ou de ressources de nature différente mais de poids ou de valeurs comparables ou reconnues comme telles par les parties impliquées ». Ces mêmes auteurs (1991: 10) opposent ce modèle à un autre qu'ils appellent "pater-nariat» dans lequel «les rapports sont inégalitaires... et le plus souvent dans un but dominant». Dans un tout autre contexte d'études menées en Nouvelle-Zélande (Park, 1992 : 582), le partenariat $n^{\prime}$ implique pas tant que les partenaires soient égaux, mais que "la relation soit celle d'un engagement commun basé sur la négociation».

Les réflexions québécoises portant sur le partenariat entre les réseaux communautaires et gouvernementaux entrevoient un climat de négociation du premier avec le second. Selon l'expérience de Guay (1991: 6), ce partenariat vise l'intégration du communautaire dans le réseau. Les pas franchis conduisent maintenant à un modèle de partenariat tripartite composé des établissements du réseau, des ressources alternatives (dans ce cas, en santé mentale) et des groupes communautaires.

Un ouvrage collectif américain consacré au «partenariat praticiens-chercheurs » en service social (McCartt Hess et Mullen, 1995) énonce comme présupposé que les praticiens peuvent jouer un rôle certain dans la construction de la connaissance. Une constante 
apparaît dans les diverses définitions du partenariat données dans cet ouvrage, soit le modèle réflexif du praticien (reflexive practitioner model) au sens où l'expérience du praticien l'amène à une « réflexion dans l'action» ou «reflection-in-action» ou "know-how does not apply». Schön (1995: 18) voit la relation entre les partenaires comme "quelque chose qui se construit sur la réflexion de telle sorte que les chercheurs puissent aider les praticiens à améliorer le processus de réflexion». Epstein (1995: 19-20) pousse encore plus loin ce paradigme en y ajoutant "les idées de la "pratique réfléchie" à tous les niveaux de la pratique (du travail social)». Pour faire le pont entre les praticiens et les chercheurs, elle recommande " $d^{\prime}$ 'incorporer, à tous les niveaux, la recherche à l'intérieur de la prise de décisions dans la pratique de manière à donner plein pouvoir aux praticiens, d'améliorer la pratique et d'éviter de compromettre principes d'action et éthique». Pour eux, "les questions viennent de la pratique et les résultats profitent à la pratique», ce qu'ils caractérisent comme étant un "practice-based research». Pour sa part, Coulton (1995: 21) étudie le partenariat à l'échelle d'une communauté locale et d'une faculté universitaire. Pour elle, "le partenariat doit être guidé par la vision, les valeurs et les questions des deux parties dans une relation d'égal à égal ». Finalement, Blum et ses associés (1995: 24) considèrent que le partenariat, en plus d'édifier des connaissances (knowledge building), oblige à faire place à l'innovation.

Bref, la plupart des auteurs recensés ne définissent pas nécessairement le partenariat. Pour certains, il est question de la nature de la relation entre les partenaires ou d'énoncés de principes. Évidemment, ces composantes renseignent sur les postulats de base et sur la philosophie qui sous-tend ce concept. Regardons maintenant de plus près ce qu'en pensent les partenaires qui ont vécu une telle expérience.

Les définitions du partenariat qui ressortent des entrevues réalisées auprès d'intervenantes de groupes de femmes, de chercheuses et de professionnelles de recherche recoupent sensiblement les idées maîtresses que l'on retrouve dans les écrits présentés précédemment. En fait, ce qui distingue le plus ces acteurs entre eux est $d^{\prime}$ abord et avant tout une question de vocabulaire plutôt que d'idées; vocabulaire qui, cela dit, contient tout de même des nuances importantes.

Ainsi, tandis que les représentantes des groupes de femmes ont parlé de partenariat en privilégiant les notions $d^{\prime}$ ' engagement» et de " complémentarité ", les chercheuses en parlent plutôt en termes de "participation commune à un projet » de recherche. II est sans doute 
inutile d'insister ici sur le fait que le terme "engagement» a une connotation beaucoup plus forte sur le plan de l'unité et de la cohésion d'une équipe que le terme participation.

Un autre aspect à signaler est le fait que les représentantes des groupes de femmes soulèvent, dans le cadre même de la définition qu'elles font du partenariat, l'importante question du partage des connaissances et des pouvoirs décisionnels; aspect, du reste qu'aucune chercheuse n'a relevé.

\section{CONDITIONS DE RÉUSSITE D'UNE EXPÉRIENCE DE PARTENARIAT}

Bien que chaque forme de partenariat soit unique et qu'il soit difficile de généraliser sans tenir compte du contexte (Park, 1992: 589), bien que le modèle à suivre doive être établi à partir de la nature du problème des personnes en cause et des ressources disponibles (Epstein et Kohn, 1992:355), certaines conditions de réussite font I'objet de constantes. Ces conditions peuvent avoir été établies comme principes ou modèles de réalisation, au préalable, ou encore être vues comme fondamentales après évaluation de l'expérience.

Pour la réussite et l'efficacité globale d'un projet de partenariat, les conditions de réussite mentionnées sont de deux ordres. D'abord, certaines sont liées aux personnes en cause, tant en ce qui concerne leur interrelation qu'en ce qui a trait à la compétence et à l'expérience professionnelle de chacune. Puis, les autres conditions ont davantage trait aux nombreux facteurs organisationnels qui interviennent à chacune des étapes de la recherche, soit au moment du choix du thème, du protocole de travail, de la qualité de la communication, de l'utilisation des résultats, du partage du temps et de l'argent investi, etc.

\section{Facteurs humains et professionnels}

Le respect entre les partenaires est retenu comme condition de réussite par toutes les études consultées, peu importe qu'elles soient québécoises, canadiennes ou américaines. En effet, il doit y avoir entre les partenaires respect mutuel, transparence, tolérance et patience (Panet-Raymond et Bourque, 1991). Ce respect peut être celui des habiletés et de l'expertise de chacun, qui sont alors considérées comme complémentaires (Black et Walther, 1995 : 155), celui des différences dans une collaboration constructive (Guay, 1991: 13; 
McCartt Hess et Mullen, 1995: 268) ou encore celui des croyances et opinions (Adams et al., 1994:6). Comme le disent Adams et al. (1994: 6), «la construction des relations est l'élément le plus important [...] sans "relationship" et une confiance établie, aucune décision, solution ou action ne peut être entreprise ». De plus, il doit y avoir une collaboration étroite entre les partenaires (Park, 1992: 591; Cummings, 1985: 290) et des rencontres d'évaluation (PanetRaymond et Bourque, 1991). Essentiellement, les personnes doivent être dans un engagement à long terme les unes envers les autres (Cummings, 1985: 304). Schecther (1988: 311) insiste aussi sur la nécessité de dépasser la perception que chaque groupe a de l'autre.

McCartt Hess et Mullen (1995: 268 et 270) signalent pour leur part la nécessité de nourrir des perspectives pluralistes et de favoriser le développement de savoirs d'expérience. Pour eux, il est également nécessaire de mobiliser des ressources servant à l'acquisition de nouvelles connaissances.

Enfin, la volonté de déléguer les tâches précises conformément aux habiletés et aux expériences propres à chacun est également considérée comme un élément de réussite (Park, 1992: 591).

\section{Facteurs organisationnels}

Une attention particulière doit être portée aux facteurs organisationnels pour qu'un projet de partenariat soit réussi. Pour n'en mentionner que quelques-uns, signalons ici un thème commun, idéalement issu des préoccupations du milieu de la pratique, un protocole de travail, des environnements interactifs, des résultats ayant une utilité pour l'intervention, une communication de qualité, un partage du temps et de l'argent. Ces facteurs sont tous jugés essentiels pour faire du partenariat une expérience réussie.

Essentiellement, les partenaires doivent se retrouver autour d'un thème commun important pour tous, soit en tant que valeur ou en tant que préoccupation (Bogo et al., 1992: 225 ; Burnette et Weiner, 1995 : 147 ; Coulton, 1995 : 120 ; Epstein et al., 1992 : 354 ; Ouellet, 1994 : 4 ; Rathbone-McCuan, Harbert et Fulton, 1991: 121; Wagner, 1991 : 481). Il est également nécessaire de créer entre les parties une situation promouvant la collaboration et l'apprentissage commun autour de ce thème (Cummings, 1985 : 299; Black et Walther, 1995 : 158).

En plus d'avoir un intérêt commun, les partenaires doivent établir ensemble ou s'entendre, à tout le moins, sur un «protocole de travail » ou un contrat. Les auteurs, bien qu'ils s'y intéressent de façon 
différente, s'entendent généralement sur la nécessité d'un tel document. Les éléments devant y être contenus varient selon les acteurs en présence. Les buts, les objectifs et les valeurs qui sous-tendent le projet, de même que les rôles et les responsabilités selon les habiletés et l'expertise de chacun, doivent toutefois être clairement définis (Colleen, Mathews et Banner, 1994 : 14; Galinsky et al., 1993 : 445; McCartt Hess et Mullen, 1995: 23; Panet-Raymond et Bourque, 1991; Park, 1992: 591; Schecther, 1988: 311-312; Colleen et al., 1994 ; Black et Walther, 1995 : 157). De cette façon, chaque partie sait ce qui est attendu d'elle. Le partage des buts communs signifie, très concrètement, que tous les collaborateurs partagent les succès et les échecs tout au long de la réalisation du projet (Black et Walther, 1995: 158).

Le protocole de travail doit aussi mettre en valeur l'engagement de l'ensemble des partenaires dans la réalisation du projet (PanetRaymond et Bourque, 1991), tout en prenant soin de leur donner une égale contribution; le praticien doit être partie prenante dès le début du projet et le dialogue entre partenaires doit être continuellement maintenu (Galinsky et al., 1993: 447; Gauthier, 1987 : 3 ; Schecther, 1988: 311). Comme le précisent McCartt Hess et Mullen (1995: 269), il faut «établir des arrangements organisationnels qui lient réellement les milieux de la pratique et ceux de la recherche». La volonté des praticiens-chercheurs de définir clairement les notions théoriques et empiriques est, selon eux, essentielle à une collaboration fructueuse (McCartt Hess et Mullen, 1995: 11). De plus, toutes les questions relatives à la diffusion de la recherche doivent aussi être clarifiées, dans le but d'assurer que les résultats seront connus dans les deux univers en cause (Cheethman, 1994: 96; Galinsky et al., 1993: 446). Des ententes quant aux produits finaux doivent donc être prises. La diffusion des résultats, par ailleurs, doit être orientée vers le milieu de la pratique et les coûts de ces activités prévus à l'étape même d'élaboration du projet (Gauthier, 1987 : 71).

De façon intéressante, l'étude québécoise de Gauthier (1987: 7-8) propose la création d' « environnements interactifs », c'est-à-dire $\mathrm{d}^{\prime}$ " endroits où chercheurs et praticiens peuvent se communiquer concepts, analyses [...] pour qu'émergent des questions et des méthodes pertinentes ». Il est donc nécessaire d'encourager des activités interactives dès l'étape de la formulation des questions de la recherche et ces dernières doivent se poursuivre tout au long de la réalisation du projet. Galinsky et ses associés (1993: 445) insistent également sur le besoin d'activités communes qui permettent de renforcer la collaboration, préparent les participants et les participantes à comprendre et à interpréter les résultats obtenus. 
Plusieurs études américaines révèlent que les résultats de la recherche doivent également être utiles et pertinents pour et dans la pratique (Bogo et al., 1992: 226; McCartt Hess et Mullen, 1995: 20; Rathbone-McCuan, Harbert et Fulton, 1991: 124; Black et Walther, 1995: 156).

Toutes les études recensées reconnaissent la qualité de la communication entre les personnes comme étant essentielle pour régler au fur et à mesure les situations litigieuses. Présente dès le début du projet, la qualité de la communication doit être continuellement maintenue tout au long du processus (Cummings, 1985: 292; Galinsky et al., 1993: 445; McCart Hess et Mullen, 1995: 7 et 268; Panet-Raymond et Bourque, 1991; Colleen et al., 1994). Une concertation est sans cesse à négocier (Couillard et Côté, 1993). De même, les projets doivent se réaliser dans un équilibre relatif sur le plan du pouvoir et la prise de décision doit être consensuelle (Adams, 1994: 6; Bogo et al., 1992: 233; Galinsky et al., 1993 : 445; McCartt Hess et Mullen, 1995: 8 et 268). Les compromis sont nécessaires et leur acceptation est inhérente au processus (Galinsky et al., 1993: 446; Colleen et al., 1994).Il est également nécessaire de normaliser les conflits, de préciser les attentes et de discuter ouvertement des différences entre partenaires. Reconnaître les problèmes et croire en la possibilité pour plusieurs acteurs de les régler est un autre préalable pour réussir l'expérience de partenariat (Colleen et al., 1994). Vus positivement, les problèmes surgissant au cours du projet peuvent être considérés comme une "occasion de consolider l'alliance » (Bogo et al., 1992: 232).

Par ailleurs, il est préférable que les groupes (ou individus) concluent des ententes quant à la question du temps à fournir et de I'argent à budgétiser (Burns et Kieschnick, 1992: 16; Park, 1992: 591). Un travail en partenariat demande un grand investissement de temps pour tous les acteurs. Aussi, pour qu'un partenariat donne des fruits, un minimum de compatibilité entre les structures est nécessaire (Couillard et Côté, 1993: 14).

Partant d'expériences concrètes de partenariat, certaines études américaines et québécoises considèrent que la collaboration doit donner lieu à des structures mixtes (Burnette et Weiner, 1995: 22). Cela peut se faire par l'entremise, notamment, d'un comité d'encadrement (Mailick et al., 1995 : 24) ou encore par la présence d'une personne intermédiaire apte à tisser le lien entre les collaborateurs (Panet-Raymond et Bourque, 1991).

Finalement, pour que le partenariat soit réussi, la ou les questions de recherche doivent émerger du milieu de la pratique, être 
issues des problèmes rencontrés par les praticiens et praticiennes (Galinsky et al., 1993: 447; Gauthier, 1987: 73 ; Gélinas et Pilon, 1994: 80). Les besoins des clients au sens large doivent devenir le but de la recherche. Dans une perspective de "praticiens-chercheurs », Schön (1995: 52) parle du praticien comme d'un "générateur» de connaissances auxquelles l'action et la réflexion donnent forme (knowing-inaction et reflecting-in-action). Epstein et Kohn (1995: 85) mettent de I'avant, quant à eux, une « research in practice» et non une « research on practice».

Les critères de réussite $d^{\prime}$ une expérience en partenariat, tels qu'ils ressortent des écrits, se situent sur un plan très général et concernent l'ensemble des acteurs qui participent à l'expérience, indistinctement de leur statut. L'analyse des cas effectuée permet toutefois de dépasser ce niveau très général et de recentrer, en fonction des différents acteurs, les principaux critères de réussite d'une telle expérience. Il est intéressant de constater, d'ailleurs, que ces critères concernent justement le rôle et les fonctions dévolus à chacun des acteurs et plus particulièrement encore les qualités que ces derniers doivent présenter. Pour faciliter la présentation de ces résultats, nous avons choisi de les regrouper en fonction des acteurs.

\section{Les caractéristiques de la représentante des groupes de femmes}

Pour les représentantes des groupes de femmes rencontrées, il semble important que la personne qui les représente dans le cadre d'une démarche de partenariat avec des chercheurs soit une personne qui se sente personnellement préoccupée par la thématique de recherche dans laquelle elle aura à s'investir. Cette personne doit aussi croire aux retombées concrètes qu'auront, sur le quotidien de l'intervention, les résultats de recherche à produire. Enfin, elle doit savoir s'exprimer avec facilité et se sentir à l'aise à l'idée de travailler avec des chercheurs. Pour ces derniers, par ailleurs, cette personne doit aussi posséder une bonne expérience du travail en équipe et dans le domaine de l'intervention, en plus de jouir d'une très grande crédibilité dans son milieu de travail.

\section{Les caractéristiques du chercheur ou de la chercheuse}

Pour garantir la réussite d'une expérience de recherche en partenariat, le chercheur ou la chercheuse, selon les représentantes des groupes de femmes, doit avoir des forces sur le plan méthodologique 
et être à même, selon les professionnelles de recherche, d'assurer une bonne direction scientifique. Les chercheuses rencontrées estiment, quant à elles, que le chercheur ou la chercheurse qui s'implique dans une démarche en partenariat doit posséder une très bonne connaissance de la problématique étudiée. Cette personne doit savoir rester collée à la réalité de l'intervention, reconnaître l'expertise de ses partenaires et ne pas travailler en fonction des seules exigences universitaires (production d'articles, etc.). Enfin, elle doit être souple et flexible dans son attitude.

\section{Les caractéristiques du professionnel ou de la professionnelle de recherche}

Selon les représentantes des groupes de femmes, pour réussir une expérience de partenariat il est nécessaire également que le professionnel ou la professionnelle de recherche engagées dans le projet possèdent des habiletés à gérer différents points de vue et soient capables d'exprimer leurs propres idées. Les professionnelles de recherche rencontrées affirment par ailleurs qu'il est nécessaire d'être diplomate et de posséder, sur le plan professionnel, une très grande rigueur scientifique.

\section{Les caractéristiques que I'on doit retrouver chez I'ensemble des acteurs}

Enfin, il semble important que l'ensemble des acteurs soient compatibles sur le plan de leur personnalité, qu'ils soient en mesure d'admettre et de reconnaître leurs compétences respectives et, enfin, qu'ils soient entièrement transparents dans leur démarche.

Donc, comme on peut le voir, les critères de réussite d'une expérience de recherche en partenariat repose en très grande partie sur les qualités des personnes engagées dans une telle démarche. Outre les qualités personnelles, la qualité de relation s'établissant entre les différents partenaires est garante, elle aussi, de la réussite ou de l'échec de cette expérience.

En prenant connaissance des écrits, il aura donc été possible de dégager, sur un premier plan, les critères généraux assurant la réussite d'une expérience en partenariat. L'analyse de quelques-unes de ces expériences aura permis, sur un second plan, de resserrer ces critères autour des différents acteurs et de dégager pour chacun d'eux les qualités particulières qu'ils devaient présenter. Nous allons maintenant discuter des obstacles au partenariat. 


\section{OBSTACLES AU PARTENARIAT}

La très grande majorité des obstacles au partenariat sont présentés dans les études consultées en référence aux deux mondes d'où proviennent les partenaires et à la difficulté de créer des ponts entre les deux. Blum et ses collaborateurs (1995: 165) ont repéré les zones principales de ces obstacles qui sont liés, soit:

- aux objectifs différents chez les partenaires,

- à une orientation divergente, résultat des différences de formation et de rôle,

- aux perceptions de la pertinence de la recherche,

- au statut prioritaire de la recherche, lorsque la responsabilité première des milieux $d^{\prime}$ 'intervention consiste à donner des services,

- à la réalisation du partenariat, incluant le système de récompenses pour les membres des milieux qui participent à la recherche,

- aux fonds disponibles et aux besoins de fonds venant de l'extérieur.

De plus, selon des études américaines, la relation entre praticiens et chercheurs est souvent caractérisée par un manque de compréhension des différences qui s'expriment par des critiques mutuelles, des conflits et un climat de compétition (Christ et Siegel, 1995 : 216). Blum et ses collègues (1995: 185) notent aussi que les sources des obstacles à la collaboration entre les agences et les universités peuvent être de trois ordres; les barrières inhérentes au partenariat agences-université, celles inhérentes à l'étape de la réalisation du changement à l'intérieur des agences ou milieux de pratique et celles qui sont propres à la forme particulière du changement. Ils font également remarquer que les expériences antérieures de partenariat qui se sont révélées mauvaises (elles étaient en réalité de la collaboration et non une relation de partenariat) compliquent les relations.

Cela dit, les difficultés nées de la rencontre de deux mondes sont aussi liées à la vision de la réalité qu'a chaque partenaire, les chercheurs étant considérés loin de la réalité et les praticiens submergés par elle (Burns et Kieschnick, 1992: 12), de même qu'à la survalorisation du savoir théorique (Burns et Kieschnick, 1992:12). Souvent, les priorités diffèrent comme la vision que chacun a de I'utilité des recherches: le praticien vise "une mise en pratique immédiate des résultats", alors que les chercheurs seraient "plus intéressés par des questions sociales à long terme explorées par la recherche»(Cheethman, 1994: 97 ; Schön, 1995: 54). 
La question du «pouvoir» entre les partenaires occasionne des relations difficiles; comme plusieurs personnes sont engagées dans un même processus, certains "contrôlent » et cette situation engendre de I'anxiété (Park, 1992: 590). Au Québec, selon Guay (1991: 8), "la culture du réseau a dominé celle du communautaire (rythme des rencontres, vocabulaire) au point de l'évacuer». Par ailleurs, face aux groupes de femmes, les universitaires ont tendance à enseigner leur savoir aux groupes, ce qui peut engendrer de la méfiance et la crainte de récupération de la part des universitaires. Les praticiens se sentent aussi évalués personnellement dans ces études (Burns et Kieschnick, 1992:12).

Des problèmes se posent aussi sur le plan des considérations d'ordre éthique. Les praticiens s'inquiètent des effets négatifs possibles de la recherche sur la pratique, par exemple, sur des clientes en crise et sur la confidentialité (Burns et Kieschnick, 1992: 12).

Finalement, les ressources financières étant limitées, les partenaires sont en compétition pour ces ressources (Burns et Kieschnick, 1992 : 11). Par contre, pour Deslauriers et Pilon (1994:31), "la crise financière et la recherche de l'efficacité exercent une pression pour une collaboration forcée. Ceci a pour conséquence que la recherche et la pratique sont prisonnières de la technocratie».

Si I'on regarde maintenant les données tirées des études de cas, les principales difficultés rencontrées dans le processus de recherche en partenariat se situent sur deux plans. Il y a d'abord les difficultés qui sont reconnues par l'ensemble des acteurs et les difficultés retenues uniquement par les représentantes des groupes de femmes.

Parmi les difficultés qu'éprouvent l'ensemble des acteurs, signalons les difficultés liées aux étapes de la recherche escamotées en raison de contraintes de temps (particulièrement en ce qui a trait au cadre d'analyse et à la clarification des mandats respectifs de toutes les participantes); de plus, on note les difficultés liées à la réalisation d'un projet de recherche lorsque l'analyse du problème ne fait pas l'objet d'un consensus entre les partenaires, ce qui entraîne des effets négatifs sur l'organisation du travail, sur l'ambiance dans laquelle se fait ce travail et sur les rapports humains.

Enfin, en ce qui concerne les difficultés qu'éprouvent plus spécifiquement les groupes de femmes, celles-ci tiennent au fait, par exemple, que les exigences de réalisation du projet de recherche sont souvent incompatibles avec les exigences du travail dans le milieu d'intervention, que le dégagement d'une travailleuse a des répercussions importantes sur l'organisation du travail et, finalement, que la non-rémunération de la participation des intervenantes pose aussi des problèmes quant à son remplacement. 


\section{DÉFIS OU GAINS À PARTICIPER}

Plusieurs gains ont été réalisés et de multiples défis demeurent. Toutefois, seuls quelques auteurs québécois se sont explicitement exprimés à ce sujet.

L'expérience de partenariat entre les ressources alternatives en santé mentale et le réseau gouvernemental constitue une amorce de changement des mentalités et une reconnaissance des ressources parallèles (Guay, 1991: 11). Pour celles-ci, il reste à «obtenir un partenariat égalitaire par la négociation à condition de renouveler les stratégies, de redessiner de nouvelles alliances et de réinventer des modes d'action collective».

En ce qui a trait au défi de l' "interface», les groupes doivent respecter leur mission, garder leur identité et leur autonomie. Le défi consiste aussi à accorder deux types de logique d'où découlent deux types de prise de décision (Couillard et Côté, 1993: 13).

Enfin, Deslauriers et Pilon (1994:32) proposent d' «éviter le discours idéaliste qui évoque des liens artificiels, mentionnant que nommer la distance entre deux pôles ne la fait pas disparaître ». Ils recommandent aussi de ne pas limiter les discussions à la méthodologie, mais d'étendre celles-ci à la politique.

Malgré les difficultés rencontrées dans le cadre des expériences de partenariat, des gains notables sont aussi liés à ce type d'expérience. Le partenariat de recherche, par exemple, participerait au processus d'empowerment de l'ensemble des acteurs; il permettrait également I'apprentissage et l'enrichissement de connaissances respectives. Ce type d'expérience, enfin, aurait des retombées positives sur l'intervention des praticiennes et sur la pédagogie des chercheurs, de même que sur les orientations pratiques de la recherche.

\section{SYNTHÈSE ET AVENUES À EXPLORER}

Pour qu'il y ait effectivement partenariat entre un groupe de recherche et un groupe communautaire, les deux parties doivent être présentes dès le début de la recherche et à chaque étape subséquente. Le rapport entre les partenaires y est égalitaire et complémentaire.

On le voit, le partenariat n'est pas une chose simple à concrétiser. Toujours présente dans les diverses études, parfois implicitement et parfois explicitement, la principale difficulté provient des deux mondes auxquels appartiennent les partenaires. Le rapport que chacun entretient avec la réalité donne une couleur particulière à sa contribution. Parfois perçue comme source de conflits, cette difficulté, si elle est dépassée, peut cependant donner lieu à une 
complémentarité riche en résultats. Le défi consiste à créer un pont entre ces deux mondes, ce que plusieurs se sont efforcés de faire.

Les conditions de réussite dont on a discuté précédemment sont autant de moyens de dépasser les obstacles mentionnés et de parvenir à un réel partenariat. Rappelons les principaux éléments. D'abord les parties doivent se retrouver autour d'une préoccupation commune quant à l'objet de la recherche. Elles doivent aussi se reconnaître comme partenaires à part entière dès le début du processus et établir conjointement les orientations du projet de recherche et de la diffusion des résultats. Par la suite, une communication constante doit être maintenue. Les conflits font partie du processus et ils peuvent resserrer l'alliance. En outre, si les partenaires sont égaux dans le travail, le pouvoir sera partagé. L'entente quant au temps et à l'argent nécessaires au projet de recherche sera alors grandement facilitée.

Deux grandes préoccupations sont manifestes: celle de la création d'un pont entre le monde de la recherche et celui de la pratique et celle de l'utilisation des résultats de la recherche. Pour plusieurs chercheurs ou groupes de travail en partenariat, les questions doivent venir de la pratique et les réponses retourner au milieu de la pratique. L'autre possibilité consiste à former les praticiens et praticiennes; ceux-ci deviennent alors des personnes possédant à la fois l'expérience du milieu d'intervention et les outils méthodologiques de la recherche. Ils peuvent ainsi formuler les questions de recherche et y apporter eux-mêmes les réponses qui prennent immédiatement vie dans le milieu. Il existe actuellement un tel programme de formation aux États-Unis, le Practitioner Research Program, en cours depuis trois ans (Cheethman, 1995: 123-137). Ce programme enseigne aux praticiens d'expérience les habiletés de base de la recherche évaluative dans un contexte d'études très circonscrites. Il se présente ainsi de nouveaux sentiers à explorer.

Un autre élément peut servir de pont: établir des «environnements interactifs » ou des activités en commun entre les partenaires, desquels peuvent émerger des questions et des méthodes pertinentes. Ces environnements interactifs peuvent se présenter sous différentes formes: des séminaires communs, la formation d'équipes ou de comités consultatifs dont le mandat est de développer des idées et des projets. Bref, autant de solutions sur lesquelles se penche actuellement le CRI-VIFF.

Le partenariat est une orientation, sinon une priorité, de plusieurs milieux de recherche et de pratique. II ne s'agit surtout pas d'occulter les conflits de divers niveaux qui peuvent se présenter tout au long de la démarche. Dans la mise en œuvre d'un partenariat, partage des valeurs et des intérêts communs et engagement mutuel sont le moteur. 


\section{NOTES}

\section{Mémo sur les auteures}

Michèle Clément est professionnelle de recherche et coordonnatrice du CRI-VIFF Québec, à I'Université Laval. Elle a été coresponsable du projet sur le partenariat.

Francine Ouellet est professeure titulaire à l'École de service social et directrice du CRI-VIFF. Elle a été coresponsable du projet sur le partenariat.

Laurette Coulombe est professionnelle de recherche, contractuelle au CRI-VIFF à I'Université Laval. Elle a participé à la rédaction de la première version de cet article.

Caroline Côté et Lucie Bélanger se sont également impliquées dans ce projet. La première a participé à la réalisation des entrevues et à la recension des écrits sur le partenariat, alors que la seconde a participé activement au comité consultatif chargé de I'orientation de ce projet.

2. Dans le cadre de ces études de cas, le CRI-VIFF a tenté de documenter des partenariats de recherche entre chercheurs universitaires et groupes de femmes dont la problématique est liée à la violence faite aux femmes. Toutefois, cette ligne directrice n'a pu être maintenue dans tous les cas, étant donné la jeunesse des expériences et surtout leur rareté.

3. Cet article plus théorique est de fait une première étape dans la réflexion sur le partenariat. Par ailleurs, dans un souci de transférabilité d'une telle réflexion, une affiche (résumant les principales questions à se poser à chacune des étapes de la recherche) a été concue comme soutien aux groupes communautaires qui souhaitent s'engager dans une démarche de partenariat de recherche. Caroline Côté, alors assistante de recherche au CRI-VIFF, et Suzanne de Guire, contractuelle à Relais-Femmes, y ont travaillé. On peut se procurer cette affiche à Relais-Femmes, 1265, rue Berri, bureau 390, Montréal (Québec) H2L 4X4, au coût de 10 \$.

4. Le Center for Research on Violence against Women and Children, de London, Ontario, est l'un des cinq centres canadiens d'excellence sur la recherche en violence, financé conjointement par Santé Canada et le CRSH, tout comme l'est le CRI-VIFF, pour le Québec.

5. Les définitions présentées dans ce texte ont fait l'objet d'une traduction libre de la part des auteures.

\section{Références bibliographiques}

Adams, B., B. Bell, K. CRAWford, D. Elias-henry, C. Hansen, A. Kytwayhat, S. Penner, J. Reid et T. WoOds (1995). The Provincial Partnership Committee on Family Violence: Final Report. Regina, Saskatchewan : Family Violence Prevention Division, Health Canada.

BLACK, R.B. et V.N. WALTHER (1995). "The Practitioner-Researcher Team: A Case Example», dans P. McCartt Hess et E.J. Mullen (dir.), PractitionerResearcher Partnerships. Building Knowledge from, in, and for Practice, p. 151-161. Washington, DC: National Association of Social Workers.

Blum, A., D.E. BieGEL, E.M. TRACY et M.J. COle (1995). "Agency-University Collaboration: Partnerships for Implementing and Studying Practice Innovations », dans dans P. McCartt Hess et E.J. Mullen (dir.), Practitioner-Researcher Partnerships. Building Knowledge from, in, and for Practice, p. 162-188. Washington, DC: National Association of Social Workers. 
Bogo, M., L. Wells, A. Sharon, A. Bergm et V. Chandler (1992). "Advancing Social Work Practice in the Health Field: A Collaborative Research Partnership », Health and Social Work, vol. 17, n 3 : 223-234.

Burnette, D. et A.S. Weiner (1995). "Intersecting the Parallel Worlds of Practice and Research », dans P. MCCARTT HESS et E.J. MULLEN (dir.), Practitioner-Researcher Partnerships. Building Knowledge from, in, and for Practice, p. 138-150. Washington, DC: National Association of Social Workers.

BURNS, N. et P. KIESCHNICK (1992). "Overcoming Barriers to the Integration of Practice and Research in the Field of Domestic Violence", Response, vol. 14, $\mathrm{n}^{\circ} 3:$ 9-17.

CheETHAM, J. (1994). "The Social Work Research Centre at the University of Stirling: A Profile», Research on Social Work Practice, vol. 1 : 89-100.

CHRIST, G.H. et K. SIEGEL (1995). "Bridging the Gap Between Practitioners and Researchers in a Hospital-Based Social Work Department », dans P. McCartt Hess et E.J. Mullen (dir.), Practitioner-Researcher Partnerships. Building Knowledge from, in, and for Practice, p. 206-226. Washington, DC: National Association of Social Workers.

CLÉMENT, M. (1993). Rapport annuel 1992-1993 du CRI-VIFF (Centre de recherche interuniversitaire sur la violence faite aux femmes et la violence familiale), 22 pages.

COlleen, R., F. MATHEWS et J. BANNer. (1994). Projet communautaire de lutte contre la violence dans les écoles: Un modèle de partenariat entre la police et l'école. Toronto: Solliciteur général du Canada, Centre de recherche.

COUILlARD, M.A. et G. CÔtÉ (1993). «Les défis d'une interface. Les groupes de femmes et le réseau de la santé et des services sociaux de la région de Québec », Service social, vol. 42, n 2 : 29-49.

COULTON, C.J. (1995). "Research for Initiatives in Low-Income Communities», dans P. McCartt Hess et E.J. Mullen (dir.), Practitioner-Researcher Partnerships. Building Knowledge from, in, and for Practice, p. 103-121. Washington, DC: National Association of Social Workers.

Cummings, T., S.A. MOHRMAN, A.M. MOHrham et G. Ledford (1985). «Organization Design for the future: A Collaborative Research Approach », dans E.E. LAWLER III (dir.), Doing Research That Is Useful for Theory and Practice, San Francisco.

Deslauriers, J.-P. et J.-M. PILON (1994). "La recherche sociale et le renouvellement des pratiques: Présentation des dossiers », Nouvelles pratiques sociales, vol. $7, \mathrm{n}^{\circ} 2: 29-33$.

EPSTEIN, I. et A. KOHN (1992). "Models of University-Agency Collaboration in Research», Research on Social Work Practice. Special Issue: Research and Practice: Bridging the Gap, vol. 2, $\mathrm{n}^{\circ} 3$.

GALINSKY, M.J., J.E. TURNBULl, D.E. MEGLIN et M.E. WiLner (1993). «Confronting the Reality of Collaborative Practice Research: Issues of Practice, Design, Measurement, and Team Development», Social Work, vol. 38, n $4: 441-449$.

GAUTHIER, F. (1987). La recherche sociale effectuée en lien avec les milieux de pratique et d'intervention: conditions favorables à sa réalisation: quatre études de cas. Québec: Conseil québécois de la recherche sociale. 
GÉLINAS, A. et J.-M. PILON (1994) «Le transfert des connaissances en recherche sociale et la transformation des pratiques sociales", Nouvelles pratiques sociales, vol. 7, $\mathrm{n}^{\circ} 2: 75-92$.

GROULX, L.-H. (1994). "Liens recherche et pratique: les thèses en présence», Nouvelles pratiques sociales, vol. 7, $\mathrm{n}^{\circ} 2: 35-49$.

GUAY, L. (1991). "Le choc des cultures: bilan de l'expérience de participation des ressources alternatives à l'élaboration des plans régionaux d'organisation de services en santé mentale», Regroupement des ressources alternatives en santé mentale du Québec (RRASMQ). Texte miméographié.

LABRECQUE, M.-F. (1994). "Un partenariat féministe de recherche : les femmes autochtones en milieu urbain.» Communication dans le cadre du colloque Formation, recherche et édition féministe à I'Université Québec, Brésil, France. Université fédérale de Rio de Janeiro.

MAILICK, M.D., D.E. BIEGEL, E.M. TRACY et M.J. COLE (1995). "Practitioners as Researcher: Development of a Collaborative Model», dans P. McCartt Hess et E.J. Mullen (dir.), Practitioner-Researcher Partnerships. Building Knowledge from, in, and for Practice, p. 189-205. Washington, DC: National Association of Social Workers.

MCCARTt Hess, P. et E.J. MULLEN (1995). Practitioner-Researcher Partnerships. Building Knowledge from, in, and for Practice. Washington, DC : National Association of Social Workers.

OUELLET, H. (1994). "Le partenariat de recherche: une orientation prometteuse des organismes subventionnaires ", Bulletin de l'ACSALF, vol. 16:4-5.

PANeT-RAYMOND, J. et D. Bourque (1991). Partenariat ou pater-nariat? La collaboration entre établissements publics et organismes communautaires œuvrant auprès des personnes âgées à domicile. Université de Montréal, Groupe de recherche en développement communautaire.

PARK, J. (1992). "Research Partnerships: A Discussion Paper Based on Case Studies From "The Place of Alcohol in the Lives of New Zealand Women" Project», Women's Studies International Forum, vol. 15, n 4: $581-591$.

RAthbONE MCCUAN, E., T.L. HARBERT et J.R. Fulton (1991). "Evaluation as an Imperative for Social Services Reservation: A Challenge to the Department of Veterans Affairs », Journal of Social Work Education, vol. 27, $\mathrm{n}^{\circ} 2: 114-124$.

SCHÖN, D.A. (1995). "Reflecting in and on Practice: A Role for Practitioners on Knowledge Building», dans P. McCartt Hess et E.J. Mullen (dir.), Practitioner-Researcher Partnerships. Building Knowledge from, in, and for Practice, p. 31-55. Washington, DC: National Association of Social Workers.

SCHECHTER, S. (1988). "Building Bridges Between Activists, Professionnals, and Researchers », dans K. Yllö et M. Bogard, Feminist Perspectives of Wife Abuse, p. 299-312. New York: Sage Publication.

WAGNER, D. (1991). "Receiving the Action Research Model: Combining Case and Cause with Dislocated Workers », Social Work, vol. 36, n ${ }^{\circ} 6$ : 465-560. 